\title{
Young Children's Use of Trait Similarity Information to Make Inference of Others
}

\author{
Seung Heon Yoo* \\ Seoul National University
}

\begin{abstract}
The purpose of this study was to understand the influence of personality trait information on young children's perception of initial attraction in peer relationships. The sample consisted of 90 children of three to five years of age in South Korea. Children were presented with an inductive inference task where they had to make inference of a target character's preference on novel-play and prosocial act based on trait labels (smart-not smart, outgoing-shy, nice-mean) and perceptual (toy) similarity information of two test characters. Children showed difference in their use of trait information depending on the perceptual similarity information, trait valence, and inference question with age. This result provides initial support that not only do young children understand the significance of trait in peer attraction but also know when trait label is more informative to use to infer others depending on the situation.
\end{abstract}

Keywords: trait label, initial attraction, perceptual similarity, young children, inductive inference

Children interact and form a relationship not only with their care-givers but also with peers as they age. According to Howes and Phillipsen (1992), young children who are enrolled in an educational setting, have at least one friend by 16-months of age and have five to six friends by the age of three. Children's circle of social interaction increases as they age and it starts to play an important role in their social and psychological development (Bagwell, Newcomb, \& Bukowski, 1998; Hymel, Vaillancourt, McDougall, \& Renshaw, 2002; Ladd \& Kochenderfer, 1996). As they attend preschool or day-care centers, children's interaction with other peers increase and they have the opportunity to choose a playmate to play with from a pool of diverse others and show

* Corresponding Author: Seung Heon Yoo, Department of Child Development and Family Studies, Seoul National University, 1 Kwanak-ro, Kwanak-gu, Seoul, South Korea. E-mail: sy64@ snu.ac.kr. preference toward a certain type of peer over others. Such preference toward a certain peer is evident even before they experience an organized educational setting by showing different interaction initiations and elicitations depending on a peer by 20 -months of age (Ross \& Lollis, 1989). The present study seeks to deepen our current understanding of young children's initial attraction in peer relationships by examining the influence of personality trait information on their perception of initial attraction among 90 children of three to five year olds in South Korea.

\section{Similarity in Peer Relationship}

Many studies have tried to understand the construct of peer relationship that children form with a certain playmate or group of playmates through looking at the similarity between friends. According to Neimeyer and Mitchell (1988), 
different types of similarity can be a predictor of initial attraction or lasting attraction between two individuals. Similarity in attitude for superficial categories (e.g., food preferences) predicted initial attraction between two individuals whereas similarities in internal qualities (e.g., personality) predicted greater attraction over time.

Studies showed that similarity in attitude (Neimeyer \& Mitchell, 1988), behaviors (Urberg, Degirmencioglu, \& Tolson, 1998), preferences (Brewer \& Silver, 1979), and values and backgrounds (Johnson, 1989) between two unacquainted individuals ignite initial attraction in adults and adolescents. Similarly, school-aged children showed initial attraction toward unacquainted peers who are similar in behavior (Haselager, Hartup, Van Lieshout, \& RiksenWalraven, 1998), attitude (Byrne \& Griffitt, 1966), and preference (Reaves \& Roberts, 1983). Even for young children, there are evidences for preference toward peers who are similar to themselves.

Consistently different studies have shown that gender plays an important role in friendship of children (Howes \& Phillipsen, 1992; La Freniere, Strayer, \& Gauthier, 1984) and children even expect others to form friends with the same gender (Martin, Fabes, Evans, \& Wyman, 1999). Howes and Phillipsen (1992) did an observational study to understand the factors that hold young children as friends. When they looked at similarity between friend pairs, they found that children usually form same-gender friends but in cross-gender friends, activity level and social interaction style were more similar compared to same-gender friends. These findings suggest that children understand other dimensions of similarity in addition to gender and can identify and prefer other peer who is similar to themselves. Similarly, Gottman (1983) showed that 3- to 9-year-old children who establish common-ground activity and explore similarity and differences of each other were more likely to become friends than those who did not. As shown in above studies, young children show preferences when choosing a friend and they tend to be friends with a peer who is similar not only in gender but also in other dimensions.

However, these data do not exactly provide information on what qualities young children are attracted to when choosing a potential playmate. Previous research on similarity of young children relied on natural observation of children interacting with others, which makes it hard to pinpoint the qualities that are important for initial attraction in young children. Such an approach also makes it unclear whether the similarityattraction effect caused the initial attraction of the relationship or the time spent together made the children similar. In response, more recent studies have tried to provide information to fill in this gap.

\section{Young Children's Attraction toward Similar Peers}

Like adults and older children, young children do show initial attraction toward others with similar preferences. In a study that examined the influence of similarity on initial attraction of 3year-old children, Fawcett and Markson (2010) showed that 3-year-old children are attracted to peers (puppets) who have similar preference of food and toy as themselves. When children were shown two puppets with different food and toy selection, they chose a puppet that made similar choice as they did. This result was also evident in 11.5-month-old infants who displayed stronger attraction toward a puppet that chose similar food choice as a playmate over a dissimilar puppet (Mahajan \& Wynn, 2012). Furthermore, in another study, 14-month-old toddlers showed initial attraction toward a third puppet that helped a puppet who showed similar food preference as themselves and a third puppet that didn't help a puppet who had dissimilar food preference (Hamil, Mahajan, Liberman, \& Wynn, 2013). These results show that young children can compare their choice with others and similarity information influences their initial attraction toward others even before they reach the age of one.

However, this does not mean that young children prefer others who are similar to 
themselves in any aspect. When children were presented with a similarity condition where the similarity factor was arbitrary (e.g., sticker, mitten) and randomly assigned by an experimenter, 3-year olds and even 11.5-monthold infants did not show preference toward a similar puppet over a dissimilar puppet (Fawcett \& Markson, 2010; Mahajan \& Wynn, 2012). This result shows that young children do not consider every kind of similarity between them and others as an important factor for initial attraction. Similarity in attitude is more important than mere superficial similarity when children consider their preference toward a peer.

The studies mentioned above have shown that similarity information plays an important role in initiating play with an unacquainted peer even for young children. However, in all of the studies, researchers looked at similarity in attitude of superficial dimensions, such as food, shirt, toy, and sticker. These similarity preferences allow us to understand that young children compare their choices with others and show initial attraction toward others who show similar preferences when given the choice of one over the other. However, questions still remain whether similarity in internal qualities like personality trait will have any influence on children's initial attraction toward a peer as a playmate, and if they consider personality trait as a factor that is influential in peer relationship of others.

\section{Children's Understanding of Personality Trait}

Previous studies have shown that children can distinguish between factors that are internal and psychological to those that are external and physical, and that internal information can be an influential factor for children when deciding whom to play with. Reaves and Roberts (1983) compared external (physique), impersonal (preference), and interpersonal (character) information to see how they affect the initial attraction of 6- to 8-year-old children toward an unacquainted peer. They found all three information to significantly influence initial attraction rating of a peer, and among the three, personality character had the strongest effect. Also, other studies have shown that young children understand personality trait and use that information to predict other's behavior and emotion.

Yuill (1992) proposed that there are two aspects to personality trait. First is behavioral regularity aspect which provides consistent behavioral information of an individual which can be used to predict the individual's future behavior. The other is causality aspect of trait which provides information of an individual's stable state of mind that generates beliefs and desires. Studies have shown that children from 4-years of age understand both aspects of trait and use trait information to predict behavior and emotion of others (Heyman \& Gelman, 1998, 1999; Yuill \& Pearson, 1998). As these studies demonstrate, young children understand both behavioral and psychological aspects of trait and they gain sufficient information about others from personality trait to predict other's behavior and emotion.

Furthermore, young children prefer to use information on similarity in trait to make inference of others' preference rather than using similarity in physical appearance information when asked to make inference of a character's nonobvious psychological preference (Heyman \& Gelman, 2000; Park \& Yi, 2007). Children from 3-years of age understand that trait label provides more information about an individual's psychological preference than mere perceptual information and prefer to use such information to infer what a target character will choose. This is why similarity in personality trait can also play a role in initial attraction toward others in young children like how similarity in preference affected their initial attraction toward an unacquainted puppet. Since young children do understand the significance of trait information in understanding others, they might use such information to evaluate other peers and use the information to choose a playmate especially in 3-, 4-, and 5-year-old children. 


\section{Children's Conception of Trait Valence}

Young children show more knowledge about positive traits and prefer them over negative traits. Studies that asked young children to predict behavior or emotion of a character in a story when the character's trait-relevant information was provided, found that children were more accurate in predicting the behavior and emotion of a character when positive traitrelevant information was provided than negative trait information (Gneep \& Chilamkurti, 1998; Koo, Ghim, Kim, Yang, Ko, \& Chung, 2006; Yuill \& Pearson, 1998). Lee and Yi (2012) also demonstrated that young children's belief in trait stability is related to the valence of trait. Young children believed that an individual described with information relevant to a negative trait, is likely to change toward being more positive with time while an individual described with a positive trait information, will stay the same over time. Such result suggests that young children are positive biased believing that people with negative trait will change toward being more positive.

In addition, Lane, Wellman, and Gelman (2013) demonstrated that young children show preference toward others who are described with a positive trait label when deciding an informant to learn from about a novel object. When provided a situation where 3-, 4-, and 5-year-old children had to choose an informant from whom they can learn about a novel object, 3-, 4-, and 5year-old children asked and endorsed an informant who were labeled with positive traits (nice, smart, and honest) than informants labeled with negative traits (mean, not smart, and dishonest). Even if the positive trait labeled informant did not have access to the relevant information, 3- and 4-year-old children still acknowledged that positive trait labeled informants are more knowledgeable. This demonstrates young children's preference toward people with positive traits even when deciding whom to learn from, and have different attitude toward different trait valence.

\section{Current Study}

The current study will try to understand young children's perception of personality trait in peer attraction through looking at their tendency to use trait similarity information to infer other's preference in novel-play and prosocial choice situations. Three, 4-, and 5-year-old children will be provided with trait label (smart-not smart, outgoing-shy, nice-mean) and perceptual (toy) similarity information of three characters and asked to make inference of a target character's preference on novel play and of prosocial act. This age groups were chosen to be studied because this is when young children start to understand that trait provides sufficient information about others and use such information to make predictions of others. Also it has been clearly shown that at least by 3 -years of age children understand their and other's preferences and show initial attraction toward others with similar preference (Fawcett \& Markson, 2010). By looking at 3-, 4-, and 5year-old children's inference of other in peer selection using trait information, young children's perception of trait information in initial attraction toward other peers will be understood and shed light to when young children start to perceive trait information as an influential factor in peer attraction.

\section{Method}

\section{Participants}

A total of ninety 3-, 4-, and 5-year-old children, 30 for each age group, were recruited from kindergarten and preschools in Seoul and Kyunggi Providence and participated in this study. The mean age for 3-year-old children was 42.5 months (range: 37-47 months), 4-year-old children was 53 months (range: 49-59 months), and 5-year-old children was 66.5 months (range: 61-71 months). 


\section{Measures}

Inductive Inference Task. Heyman and Gelman's triad inference task (2000) was used after being modified to make it more suitable for the purpose of the study. Participants were presented with a slide of triad line-drawn characters ( 2 test characters and 1 target character) using Microsoft PowerPoint, and each character was described using trait similarity information (contrastive trait label pair) while pointing to each corresponding character (e.g., "This child is nice", "This child is mean", "This child is nice"). Then same triad line-drawn characters slide was shown but this time with perceptual similarity information (stick/block) added to the picture, and perceptual similarity information was described to the participants in the similar way as the trait information was described (e.g., "This child has a stick", "This child has a block", "This child has a block").

These similarity information were pitted against each other so that if one of the test characters shared one similarity information with the target character, it would possess a dissimilar quality for the other similarity information with the target character. Thus, two test characters would have different traits and perceptual similarity qualities but share one similarity quality with the target character. For example, one of the test characters would have a similar toy as the target character but be described with a dissimilar trait label, and the other test character would have a dissimilar toy but be described with a similar trait label as the target character.

After both similarity information were presented to the participants, they were asked a memory test question to see if they remembered the trait label of the characters (e.g., "Who is mean/nice?"). For those who did not remember the trait label of the characters, each character's trait similarity information was retold. If the participant chose the right character, the researcher moved on to the next stage of the study.

Next, novel-play preference information was described for each test character while pointing to the corresponding character (e.g., "This child likes 'Tibit' play", "This child likes 'Momo' play"). Novel-plays were used to make sure that the participants didn't have any prior knowledge of the play. This insured that the participants only used similarity information of the characters to make inference of the target character's preference for a novel play. Then the children were asked a forced question to infer which novel play the target character would like to play (e.g., "Will this child (target character) like 'Tibit' play as this child (test child 1) does or 'Momo' play as this child (test child 2) does?"). After the participants made their choice, they were asked another memory question to check if they remembered the trait of the characters. Finally, the participants were asked to make inference of who the target character would choose to help if both test characters were in need of help ("These two children (test characters) need help. Who do you think this child (target character) would help?").

The inference choices that the participants made were either scored as 0 or 1 for both novelplay and prosocial questions. If their choice was inferred from trait similarity information (choosing a test character who had same trait label), they received a score of 1 . On the other hand, if the participant inferred from perceptual similarity information (choosing a test character who had similar toy or skin color), they received a score of 0 . Thus, the total score the participants received from the inference task referred to the score they received for making inference based on trait similarity information, and higher the score, the more they inferred from trait information.

Inference Questions. Two inference questions were asked to the participants. One was on novel-play that the target character would like to play. A novel-play inference question was asked because playstyle is related to formation of friendship in children (Gottman, 1983). Without knowing what the novel-play is, participants have to make inference based on the similarity information of the target and test characters. Since children prefer to play with other peers who share similar playstyle, participants' choice 
of this inference question will provide an initial evidence of how they think trait label is related to play and thus to peer attraction.

The other question was on prosocial choice that the target character would make. Prosocial question was asked because children show greater prosocial inclination toward ingroup members since early in life (Moore, 2009; Young, Fox, \& Zahn-Waxler, 1999). So by asking the prosocial question, children's understanding of the relationship between trait and prosocial act can be observed.

\section{Procedure}

Pilot Study. After completing the research design, a total of three pilot studies were conducted to find out if the research tool is applicable to young children.

The first pilot study was conducted to select three contrasting trait label pairs from a pool of trait labels that were used by studies that looked at children between the ages of 3 to 5 . Three graduate students in Child Studies, who also had experience of teaching young children at a preschool, were asked to choose 5 contrasting pairs from the pool of trait labels that 3- to 5year-old children use and understand, and were asked to revise the trait labels so that they can be more familiar to children. Then, three 3-, 4-, and 5 -year-old children each were individually asked for their understanding of the trait labels ("Do you know what OOO is?") and if they had a prior experience with the trait labels ("Do you know a person who is OOO?"). Through the process, three contrasting pairs of trait labels were selected for the study: nice-mean, outgoing-shy, and smart-not smart.

The second pilot study was conducted on 4 children of each age group. The initial research design of the current study was similar to Heyman and Welman's study (2000) in that the picture of triad line-drawn characters always included perceptual similarity information, and provided trait similarity information of each character every time new information (perception similarity and novel play) was described and when the inference question was asked. However, it took about 35 to 45 minutes for the participants to complete the whole task and made it hard for them to concentrate. Thus, the research design was revised to describe the trait similarity information only once but included two memory tests to check to see if the participants remembered the trait information of the characters. In addition, to help the participants to focus while the trait information were being described, a triad picture with no information of perceptual similarity was presented when trait information was described rather than using a triad picture which always included perceptual similarity information as it initially did.

The final pilot test was conducted on 4 children of each age group, and even 3-year-old children were able to use trait labels to identity two characters as similar by saying "They are both nice". It took about 25 minutes for 4- and 5year-old children and 30 minutes for 3-year-old children to complete the whole task. To help children to concentrate on the task, it was divided into two sessions (12 individual tasks each) and each session was conducted on a separate day.

Present Study ${ }^{l}$. The data was collected from two preschools and one kindergarten located in Seoul and Kyunggi Providence. Researcher and one other graduate student interviewed each participant in a separate room located at the preschool or kindergarten. When a participant entered the room, s/he was greeted by a researcher and was sat next to the researcher facing a screen. After asking for the child's name and the class $\mathrm{s} /$ he is in, the researcher spent about a minute to talk with the participant on any topic to familiarize the participant to the researcher. When the participant looked comfortable, s/he was introduced to the task $\mathrm{s} / \mathrm{he}$ was about to perform. When the child was ready to start, the triad characters were shown on the screen and the task started. Children answered either verbally or by pointing with their figures. When the children answered, their answers were

1 This study was approved by SNU IRB committee (IRB No. 1401/001-002) 
recorded on a separate score sheet. When the participants completed 12 tasks, they were told that they did an excellent job and was provided with a gift. Each session took about 10 to 15 minutes for the participants to complete.

\section{Results}

Children's use of trait labels to make inference of target character's preference was examined by asking two inference questions to see if their use of trait similarity information will differ by perceptual information and inference questions. In order to understand children's use of trait labels by age, trait valence, and perceptual similarity information, repeated measures ANOVA was used. Trait valence and perceptual similarity information were entered as within factors, age as between factor, and trait label scores as the dependent variable.

\section{Novel-play Inference Task}

Interaction effects of age and trait were shown in use of trait labels when children were asked to make inference of a target character's preference for a novel-play with perceptual information of toy as shown in Table 1.

When interaction effect of age was looked at, there was a significant difference in children's trait label scores by age $(F=4.06, d f=2,87$, $p<.05)$. Post-hoc test using Bonferroni showed significant difference between 3- and 5-year-old children but showed no difference between 3and 4-year-old children and 4- and 5-year-old children. This result shows that trait similarity information is used more by 5 -year-old children than 3-year-old children to make inference of others in novel-play task. This result is inconsistent with Park and Yi's result (2007) which showed 4- and 5-year-old children using significantly more trait label information to infer others than 3-year-old children. This discrepancy could be related to the inference question that was asked in the current study.

In Park and Yi's study (2007), the inference questions were not directly related to the perceptual information. For example, trait label and facial appearance information were used to describe the characters but the inference questions were about target character's preferences for issues that were unrelated to appearance like favorite TV show and teacher.

Table 1

Repeated Measures ANOVA on Young Children's Trait Label Scores by Age, Trait, and Toy in Novel-play Inference Task

\begin{tabular}{|c|c|c|c|c|c|c|c|}
\hline & Source & & $\begin{array}{l}\text { Sum of } \\
\text { Squares }\end{array}$ & $d f$ & $\begin{array}{l}\text { Mean } \\
\text { Square }\end{array}$ & $F$ & $\begin{array}{c}\text { Post-hoc Test } \\
\text { (Bonferroni) }\end{array}$ \\
\hline \multirow{11}{*}{$\begin{array}{l}\text { Trait } \\
\text { Label } \\
\text { Score }\end{array}$} & \multirow[t]{2}{*}{ Within } & Age & 10.27 & 2 & 5.14 & $4.06^{*}$ & $\mathrm{a}<\mathrm{c}$ \\
\hline & & Std. Error & 109.97 & 87 & 1.26 & & \\
\hline & \multirow[t]{9}{*}{ Between } & Trait & 5.14 & 1 & 5.14 & $5.67 *$ & $\mathrm{~d}<\mathrm{e}$ \\
\hline & & Trait x Age & 4.27 & 2 & 2.14 & 2.36 & \\
\hline & & Std. Error & 78.84 & 87 & .91 & & \\
\hline & & Toy & .23 & 1 & .23 & .37 & \\
\hline & & Toy x Age & .45 & 2 & .23 & .37 & \\
\hline & & Std. Error & 53.58 & 87 & .62 & & \\
\hline & & Trait x Toy & .34 & 1 & .34 & .81 & \\
\hline & & Trait x Toy x Age & 1.81 & 2 & .90 & 2.18 & \\
\hline & & Std. Error & 36.11 & 87 & .42 & & \\
\hline
\end{tabular}

$* p<.05$

Note: $\mathrm{a}=3, \mathrm{c}=5, \mathrm{~d}=$ negative trait, $\mathrm{e}=$ positive trait 
On the contrary, in the current study, toy similarity information was directly related to the inference question of novel-play. Even though young children did not have prior knowledge of what the novel-play is, they might have linked the relationship between the term 'play' with toy through their daily experiences of playing with toys.

Similarly, Heyman and Gelman (2000) showed that when there is a direct relationship between information used to describe characters and the inference question, 3- and 4-year-old children use information that is related to the inference question to make their inferences (e.g., using facial appearance and asking inference question that includes phrase like 'looks alike'). So, children in this study might have associated toy with novel-play to make their inference of a target character's preference for a novel-play, and the reason why there was no significant difference in the trait label scores between 3- and 4-year-old children might be due to their perceptual dependence to the toy similarity information presented. Their stereotypic view of the relationship between toy and play has made them to use the perceptual information to infer other's preference for a play similar to Hoffner and Cantor's result (1985) where children related to perceptual information of an old lady (generous/mean looking) to predict her behavior toward intruders rather than her past behavior (nice or mean). On the bases of this explanation, it could be presumed that 5-year-old children start to understand trait label as an influential source of information when inferring a playstyle and thus might recognize trait similarity as a factor that initially attracts peers when deciding a play.

When interaction effect of trait was looked at, there was a significant difference in children's trait label scores depending on the valence of trait $(F=5.67, d f=1,87, p<.05)$. Post-hoc test using Bonferroni showed that when the trait labels were positive, children's trait label scores were significantly higher than the scores for negative trait labels. This result shows that young children tend to use more trait label similarity information when trait labels are positive to make inference of other peer's choice on novel-play than when trait labels are negative. This result coincides with a previous study that showed older children's preference toward individuals with positive than negative personality character when choosing a peer to play with (Reaves \& Roberts, 1983).

\section{Prosocial Inference Task}

Interaction effects of age and trait were shown in use of trait labels when children were asked to make inference of a target character's preference for a prosocial act with perceptual information of toy as shown in Table 2.

When interaction effect of age was looked at, there was a significant difference in children's trait label scores by age $(F=4.23, d f=2,87$, $p<.05)$. Post-hoc test using Bonferroni showed significant difference between 3- and 4-year-old children and 3- and 5-year-old children but showed no significant difference between 4- and 5-year-old children. So 4- and 5-year-old children used significantly more trait labels than 3-year-old children when making inference of whom the target character would help. This finding is consistent with previous study which has found that 4- and 5-year-old children use trait label information significantly more than 3year-old children to infer others (Park \& Yi, 2007). This shows that from 4-years age, children realize peers with similar trait will help each other and show ingroup preference toward others with similar trait label.

However, different from novel-play inference task, 4- and 5-year-old children used trait similarity information significantly more than 3year-old children to infer other's prosocial choice. One factor that prevented 3- and 4-yearold children from using trait information was the toy perceptual information in the novel-play inference task. So another possible explanation for the above result is that 3-year-old children are more perceptually dependent on toy than 4- and 5 -year-old children with their trait label score being lower than 4- and 5-year-old children's scores even with prosocial inference question.

Three-year-old children have been found to 
Table 2

Repeated Measures ANOVA on Young Children's Trait Label Scores by Age, Trait, and Toy in Prosocial Inference Task

\begin{tabular}{|c|c|c|c|c|c|c|c|}
\hline & Source & & $\begin{array}{l}\text { Sum of } \\
\text { Squares }\end{array}$ & $d f$ & $\begin{array}{l}\text { Mean } \\
\text { Square }\end{array}$ & $F$ & $\begin{array}{c}\text { Post-hoc Test } \\
\text { (Bonferroni) }\end{array}$ \\
\hline \multirow{11}{*}{$\begin{array}{l}\text { Trait } \\
\text { Label } \\
\text { Score }\end{array}$} & \multirow[t]{2}{*}{ Within } & Age & 10.52 & 2 & 5.26 & $4.23 *$ & $\mathrm{a}<\mathrm{b}, \mathrm{c}$ \\
\hline & & Std. Error & 108.21 & 87 & 1.24 & & \\
\hline & \multirow[t]{9}{*}{ Between } & Trait & 6.67 & 1 & 6.67 & $5.91 *$ & $\mathrm{~d}<\mathrm{e}$ \\
\hline & & Trait x Age & 1.94 & 2 & .97 & .86 & \\
\hline & & Std. Error & 98.14 & 87 & 1.13 & & \\
\hline & & Toy & 2.67 & 1 & 2.67 & 2.81 & \\
\hline & & Toy $\mathrm{x}$ Age & .34 & 2 & .17 & .18 & \\
\hline & & Std. Error & 82.74 & 87 & .95 & & \\
\hline & & Trait x Toy & .47 & 1 & .47 & .91 & \\
\hline & & Trait $x$ Toy $x$ Age & 2.61 & 2 & 1.30 & 2.54 & \\
\hline & & Std. Error & 44.68 & 87 & .51 & & \\
\hline
\end{tabular}

$* p<.05$

Note: $a=3, b=4, c=5: d=$ negative trait. $e=$ positive trait

prefer others who choose similar food, toy, and hair-color as themselves but among these similarities, toy was the most influential source of information that triggered initial attraction toward an unacquainted peer (Fawcett \& Markson, 2010). Likewise, in the current study, 3 -year-old children might have preferred to infer other's preference by using toy similarity information since toy might be more influential source of information than trait in initial attraction even for prosocial acts. Thus, this suggests that 3-year-old children are perceptually dependent on the perceptual information of toy even for inference questions that are not directly related to toy.

When interaction effect of trait was looked at, there was a significant difference in children's use of trait labels by trait valence $(F=5.91, d f=1$, $87, p<.05)$. Post-hoc test using Bonferroni showed that young children's trait label score was significantly higher when the trait labels were positive than negative. This result is consistent with the novel-play inference task showing that young children use more trait similarity information to make inference of others when trait labels are positive than negative.
As mentioned in the novel-play inference section, this result coincides with previous studies that showed that young children prefer positive traits over negative traits.

\section{Discussion}

Similar to previous studies which showed that children from 4-years of age understand and use trait labels to infer other's preferences (Park \& Yi, 2007), this study also showed that children from 4-years of age use trait similarity information to infer other's preference on novelplay and of whom to help. However, there was difference in how children used trait similarity information depending on the perceptual similarity information and trait valence with age.

Young children were affected by perceptual information of toy when they were asked to make inference of a target character's preference for a novel-play. Only 5-year-old children showed significant difference in their use of trait information from 3-year-old children and there was no significant difference between 4- and 3year-old children's use of trait information. This 
result was different from Park and Yi's result (2007) which showed 4- and 5-year-old children using significantly more trait information than 3year-old children to infer other's nonobvious psychological preferences. This difference could be due to 3- and 4-year-old children's perceptual dependence to toy when inferring a target character's preference for a novel-play. They might have relied more on their experience of using toys for plays and such stereotypic view of toy might have affected their choice of information to make their inference rather than using conceptual relationship between trait and playstyle which seemed to be the case for 5-yearold children. This is similar to Hoffner and Cantor's study (1985) where children relied on perceptual appearance of an old lady to determine her actions toward intruders rather than using information of her past behavior. So, for perceptual information of toy with novel-play inference question, 3- and 4-year-old children relied more on the perceptual similarity information to make their inference whereas 5year-old children used more trait similarity information to make their inferences. This result suggests that from 5-years of age, children begin to recognize trait as an influential factor in determining other peer's preference for a play and show initial attraction toward others who are similar in trait.

However, 4-year-old children also showed their ability to use trait similarity information to infer others with perceptual similarity information of toy when inference question was not directly related to toy. When children were asked to infer other's choice of prosocial act with perceptual information of toy, both 4- and 5year-old children showed significant difference in their use of trait labels from 3-year-old children. This demonstrates that 4-year-old children's perceptual dependency on toys was only shown when inference question is directly related to the perceptual information of toy, and when there is no clear relationship between these two factors, even 4-year-old children use significantly more trait information to make their inferences than 3-year-old children. However, 3year-old children continued to show perceptual dependence to toy even when there is no clear relationship to the inference question. This suggests that toy is an important information for 3 -year-old children in their preference toward an unacquainted peer and similar finding was shown in Fawcett and Markson's study (2010) where 3-year-old children's initial attraction toward a peer was most affected by similarity in toy preference.

So the current study demonstrated young children's gradual change of information usage from being perceptual to being more of conceptual users of information to infer other's preference with perceptual information of toy, suggesting that young children do consider conceptual information like trait when considering an unacquainted peer as a playmate. Also it showed that at least by 4-years of age, children consider the information they have acquired to use the most informative source of information to decide their preference toward other peers and trait is one of the influential information that they consider in deciding their attraction toward unacquainted peers.

Another factor that significantly affected young children's use of trait similarity information was trait valence. In all of inference tasks, children used significantly more trait information to infer other's preferences when trait labels were positive than negative. Children showed preference toward positive traits, and their decision to use trait information was determined by the valence of trait. In both inference tasks, children started to show inclination toward similar positive traits to infer others and their use of trait information significantly diminished with negative traits. This could be due to the perception that people have on the valence of traits, believing that positive traits are more socially acceptable than negative traits, and might have affected the children's view of trait. This preference toward positive traits could be the beginning for children to show preference over peers who have positive personality characters as was shown in a previous study which showed positive character to be the most influential predictor for older children's initial attraction toward an 
unacquainted peer (Reaves \& Roberts, 1983).

To conclude, the current study showed that at least by 5 -years of age and possibly by age 4 , children realize that trait similarity is an informative source of information in initial attraction between peers. The results of this study suggest that children at this age are aware that peers with similar traits share similar preferences toward a play and also peers tend to help others who are similar in traits. However, their use of trait information is influenced by the perceptual similarity information and inference questions. Also, young children are affected by the valence of trait when using trait information. This is an initial evidence that even children as young as 5-years of age understand that personality traits play a role in how peers view others and can influence initial attraction toward peers.

\section{References}

Brewer, M. B., \& Silver, M. (1978). Ingroup bias as a function of task characteristics. European Journal of Social Psychology, 8, 393-400.

Byrne, D., \& Griffitt, W. (1966). A developmental investigation of the law of attraction. Journal of Personality and Social Psychology, 4, 699-702.

Fawcett, C. A., \& Markson, L. (2010). Similarity predicts liking in 3-year-old children. Journal of Experimental Child Psychology, 105, 345358.

Gneep, J., \& Chilamkurti, C. (1988). Children's use of personality attributions to predict other people's emotional and behavioral reactions. Child Development, 59, 743-754.

Gottman, J. M. (1983). How children become friends. Monographs of the Society for Research in Child Development, 48, 1-86.

Hamlin, J. K., Mahajan, N., Liberman, Z., \& Wynn, K. (2013). Not like me = bad: Infants prefer those who harm dissimilar other. Psychological Science, 24, 589-594.

Haselager, G. J., Hartup, W. W., Van Lieshout, C. F., \& Riksen-Walraven, J. M. (1998). Similarities between friends and nonfriends in middle childhood. Child Development, 69, 1198-1208.

Heyman, G. D., \& Gelman, S. A. (1998). Young children use motive information to make trait inferences. Developmental Psychology, 34, 310-321.

Heyman, G. D., \& Gelman, S. A. (1999). The use of trait labels in making psychological inferences. Child Development, 70, 604-619.

Heyman, G. D., \& Gelman, S. A. (2000). Preschool children's use of trait labels to make inductive inferences. Journal of Experimental Child Psychology, 77, 1-19.

Hoffner, C., \& Cantor, J. (1985). Developmental differences in responses to a television character's appearance and behavior. Developmental Psychology, 21, 1065-1074.

Howes, C., \& Phillipsen, L. (1992). Gender and friendship: Relationships within peer groups of young children. Social Development, 1, 230-242.

Hymel, S., Vaillancourt, T., McDougall, P., \& Renshaw, P. D. (2002). Peer acceptance and rejection in childhood. In P. K. Smith \& C. H. Hart (Eds.), Blackwell handbook of childhood social development (pp. 265-284). Oxford, UK: Blackwell.

Johnson, M. A. (1989). Variables associated with friendship in an adult-population. Journal of Social Psychology, 129, 379-390.

Koo, J., Ghim, H., Kim, K., Yang, H., Ko, S., \& Chung, M. (2006). Children's understanding of personality traits: With focus on trait inference and situational and temporal stability [아동의 성격 특질에 대한 이해 발달: 특질추론과 상황적, 시간적 안정성을 중심으로]. The Korean Journal of Developmental Psychology, 19, 1-20.

Ladd, G. W., \& Kochenderfer, B. J. (1996). Linkages between friendship and adjustment during early school transition. In W. M. Bukowski, A. F. Newcomb, \& W. W. Hartup (Eds.), The company they keep: Friendship in childhood and adolescence (pp. 322-3450). New York: Cambridge University Press.

La Freniere, P., Strayer, F. F., \& Gauthier, R. (1984). The emergence of same-sex affiliative preferences among preschool peers: A 
developmental/ ethological perspective. Child Development, 55, 1958-1965.

Lane, J. D., Wellman, H. M., \& Gelman, S. A. (2013). Informant's trait weigh heavily in young children's trust in testimony and in their epistemic inferences. Child Development, 84, 1253-1268.

Lee, J. H., \& Yi, S. (2012). 3-, 4-, 5-year-old children's beliefs about trait stability based on trait type and valence [3, 4, 5 세 유아의 특질 유형과 정서에 따른 특질 안정성에 대한 믿음]. Korean Journal of Human Ecology, 21, 83-93.

Mahajan, N., \& Wynn, K. (2012). Origins of us versus them: Prelinguistic infants prefer similar others. Cognition, 124, 227-233.

Martin, C. L., Fabes, R. A., Evans, S. M., \& Wyman, H. (1999). Social cognition on the playground: Children's belief about playing with girls versus boys and their relations to sex segregated play. Journal of Social and Personal Relationships, 16, 751-771.

Moore, C. (2009). Fairness in children's resource allocation depends on the recipient. Psychological Science, 20, 944-948.

Neimeyer, R. A., \& Mitchell, K. A. (1988). Similarity and attraction: A longitudinal study. Journal of Social and Personal Relationships, 5, 131-148

Park, Y., \& Yi, S. (2007). Preschoolers' use of trait labels to make inferences about people according to their age and task variables $[3,4$, 5 세 유아의 과제 변수에 따른 대인 추론: 특질단서 사용을 중심으로]. The Korean Journal of Developmental Psychology, 20, 7794.
Reaves, J. Y., \& Roberts, A. (1983). The effect of type of information on children's attraction to peers. Child Development, 54, 1024-1031.

Ross, H. S., \& Lollis, S. P. (1989). A socialrelations analysis of toddler peer relationships. Child Development, 60, 1082-1091.

Urberg, K. A., Degirmencioglu, S. M., \& Tolson, J. M. (1998). Adolescent friendship selection and termination: The role of similarity. Journal of Social and Personal Relationships, 15, 703-710.

Young, S. K., Fox, N. A., \& Zahn-Waxler, C. (1999). The relations between temperament and empathy in 2-year-olds. Developmental Psychology, 35, 1189-1197.

Yuill, N. (1992a). Children's conception of personality traits. Human Development, 35, 265-279.

Yuill, N., \& Pearson, A. (1998). The development of bases for trait attribution: Children's understanding of traits as causal mechanisms based on desire. Developmental Psychology, 34, 574-586. 